\title{
Prevalência de Burnout em médicos de um Hospital Público de São Paulo
}

\author{
Prevalence of Burnout in public hospital \\ doctors in São Paulo
}

Renato Arimateia Costa Magalhães ${ }^{1}$, Débora Miriam Raab Glina²

Magalhães RAC, Glina DMR. Prevalência de Burnout em médicos de um Hospital Público de São Paulo. Saúde, Ética \& Justiça. 2006;11(1/2):29-35.

RESUMO: Objetivo: Verificar a prevalência da Síndrome de Burnout em médicos de um Hospital Público na cidade de São Paulo. Métodos. Executou-se um estudo de caso em um Hospital Geral Público. Trata-se de um estudo transversal com a aplicação do Maslach Burnout Inventory (MBI) e questionário sócio-demografico em 76 médicos plantonistas que representam cerca de $25 \%$ da população total. O MBI é um questionário auto aplicado composto de três escalas: exaustão emocional (EE), realização pessoal (RP) e despersonalização (DE). Resultados e Discussão. Foi encontrado um índice de $11 \%$ dos médicos com Síndrome de Burnout que estão dispostos do seguinte modo: $47 \%$ dos casos estão na faixa etária dos 31 aos 45 anos; o sexo feminino representa $52 \%$ dos casos; os casados representam $67 \%$; já a população com Burnout que não possui filhos representa $67 \%, 100 \%$ realizaram alguma especialização após a graduação e 34\% estão no período que corresponde de 11 a 25 anos de formado; na questão "cargo" ou função os casos de Burnout estão distribuídos da seguinte forma:anestesistas com $25 \%$ dos casos, cirurgiões com $25 \%$, pediatras $25 \%$, ortopedistas e clínicos com $12,5 \%$ cada. Dos médicos ocupantes dos cargos acima descritos, $62 \%$ trabalham no pronto socorro e $37,5 \%$ em outros setores do hospital; $62,5 \%$ deles trabalham mais de 61 horas semanais e $100 \%$ possui mais de 03 empregos. Considerações finais. Este estudo demonstrou que, uma pequena parcela dos médicos (11\%) apresentou a doença com todas as suas características (EE e DE elevados e PP baixos), contudo, uma grande porcentagem de médicos está a ponto de apresentar a síndrome, dado os elevados índices de exaustão emocional (EE) e despersonalização (DE) (perto de $80 \%$ entre moderado e alto), o que só não ocorreu ainda devido à grande realização pessoal na profissão.

DESCRITORES: Médicos hospitalares. Médicos/psicologia. Estresse psicológico/prevenção \& controle. Condições de trabalho. Local de trabalho/psicologia.

\footnotetext{
1 Médico, especialista em Medicina do Trabalho. Departamento de Medicina Legal, Ética Médica e Medicina Social e do Trabalho da Faculdade de Medicina da Universidade de São Paulo (USP).

2 Psicóloga, Doutora em Psicologia Social e Ergonomista. Departamento de Medicina Legal, Ética Médica e Medicina Social e do Trabalho da Faculdade de Medicina da Universidade de São Paulo (USP).

Endereço para correspondência: Débora Miriam Raab Glina. Rua Teodoro Sampaio, 115. CEP 05405-000. São Paulo, SP. e-mail: deboraglina@uol.com.br
} 


\section{INTRODUÇÃO}

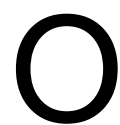
relacionamento entre médicos e pacientes assim como entre médicos e organizações públicas ou privadas está se tornando cada vez mais complexo. De um lado, os médicos têm sido vistos com desconfiança tanto pelos pacientes como pelos empregadores públicos e privados. Por outro lado, os médicos mostram-se insatisfeitos e estressados.

Ferreira $^{9}$ define estresse como sendo o conjunto de reações do organismo a agressões de ordem física, psíquica, infecciosa, e outras, capazes de perturbar-Ihe a homeostase (homeostase: tendência à estabilidade do meio interno do organismo).

O tipo de estresse crônico que consome o trabalhador física e emocionalmente, deixando-o agressivo e irritadiço e/ou desmotivado, desinteressado, causando um mal-estar interno, insatisfação ocupacional e uma insensibilidade com relação a quase tudo e todos é definido como Síndrome de Burnout.

O conceito Burnout foi lançado por Freudenberger em 1975, sendo uma síndrome do esgotamento, com foco principal na exaustão emocional, na fadiga e na frustração, decorrentes do desgaste ou da não satisfação em relação à profissão.

Acomete em grande parte profissionais ligados a saúde ou profissões de ajuda ao próximo (médicos, enfermeiros, professores, etc.), também os perfeccionistas, os idealistas e os profissionais pouco reconhecidos ${ }^{10,11}$

A Síndrome de Burnout na concepção de Maslach (1982) apud Glina ${ }^{10}$, é ainda caracterizada por três aspectos básicos:

$>$ A exaustão emocional: sentir-se drenado, sugado pelos outros e pelo trabalho. Devido a esta intensa carga emocional o profissional, sente-se incapaz de repor as energias para um próximo dia de trabalho, tornando-se assim intolerante, nervoso e amargo, tanto no ambiente de trabalho como fora dele;

$>$ a despersonalização: é o desenvolvimento de atitudes e sentimentos negativos, insensíveis e de falta de preocupação com pessoas que são receptoras de seus serviços; torna o individuo "desumano";

$>$ e, o sentimento de reduzida realização e satisfação pessoal e profissional: traduz a diminuição nos sentimentos de competência e realização no trabalho com pessoas. Um exemplo disso é a classe médica que espera ter algum tipo de retribuição positiva das pessoas, uma vez que desenvolve um papel na busca do bem-estar destas, o que nem sempre acontece e em virtude desta exaustão emocional de expectativas frustradas associadas a precárias condições de trabalho, não é raro surgirem sentimentos de decepção, queda da auto-estima, desinteresse, podendo chegar até mesmo a depressão ${ }^{10,11}$

Por essas razões o Burnout não se confunde com tédio, alienação, ansiedade, insatisfação no trabalho, fadiga, depressão ou estresse. Sua distinção no âmbito das doenças mentais em trabalhadores está bem definida, sendo delimitado pelos aspectos acima.

A síndrome de Burnout tem sido defendida entre diversos autores como sendo diferente do estresse, pois o Burnout tem reflexo direto sobre clientes, pacientes e pessoas em geral que se relacionam com o individuo acometido por esse mal, já o estresse reflete-se sobre o próprio individuo e sua vida, não envolvendo sua relação com o trabalho².

Além disso, o Burnout caracteriza-se pelas ausências ao trabalho e realização de tarefas de forma inadequada, bem como o trato ríspido para com os outros. Tem sido considerado como a maior causa de absenteísmo em profissionais da saúde atualmente.

Os distúrbios mentais estão definidos como os diagnósticos correspondentes ao capítulo $\mathrm{F}$ do Código Internacional de Doenças (CID - 10) $)^{15}$, mais especificamente, a Síndrome do Esgotamento Profissional ou Burnout se encontra definida em ( $Z$ 73.0) e no Protocolo de Procedimentos MédicoPericiais N5, XII, que a denomina como: Sensação de estar acabado, definindo-a como

"uma reação à tensão emocional crônica gerada a partir do contato direto e excessivo com outros seres humanos, particularmente quando estes estão preocupados ou com problemas, em situações de trabalho que exigem tensão emocional e atenção constantes, e grandes responsabilidades"13.

A relação da "Síndrome de Burnouf" com o trabalho, poderá estar vinculada aos seguintes fatores que influenciam o estado de saúde:

$>$ "Ritmo de trabalho penoso" (Z 56.3);

$>$ "Outras dificuldades físicas e mentais relacionadas com o trabalho" ( $\mathrm{Z} 56.6)$.

Portanto, havendo evidências epidemiológicas da incidência da Síndrome em determinados grupos ocupacionais, sua ocorrência em trabalhadores destes grupos poderá ser classificada como "doença relacionada com o trabalho", do Grupo II da Classificação de Schilling, posto que o "trabalho" ou a "ocupação" podem ser considerados como fatores 
de risco, no conjunto de fatores de risco associados com a etiologia multicausal desta doença.

Entre as profissões de saúde, o trabalho do médico tem sido muito estudado do ponto de vista psicológico e sociológico. Essa profissão está diretamente ligada à vida de outros seres humanos, suscitando sentimentos muito fortes e contraditórios como: piedade, compaixão e amor; culpa e ansiedade; ódio e ressentimento, contra seus pacientes ou até mesmo contra os familiares destes. Em contrapartida os pacientes e seus parentes demonstram apreço, gratidão, afeição, respeito; ou ainda podem ser exigentes, possessivos, ciumentos, ingratos, desbocados, agredindo muitas vezes os profissionais da saúde no contexto geral.

Há inúmeras gratificações psicológicas inerentes à profissão médica. Aliviar a dor e o sofrimento, curar doenças, salvar vidas, diagnosticar corretamente, sentir-se competente, ensinar, aconselhar, educar, prevenir doenças, receber reconhecimento e gratidão são algumas das características psicológicas da tarefa médica que fazem da medicina uma profissão ainda muito atraente e gratificante.

Há no trabalho clínico, a exposição a poderosas "radiações" psicológicas emanadas do contato íntimo com o adoecer, pois no âmbito assistencial dos serviços de emergência, ocorrem situações tão dramáticas como talvez em nenhum outro campo da atividade humana em tempos de paz.

Este caráter estressante inerente à tarefa médica tem se amplificado significativamente devido ao volume de pacientes e às precárias condições de trabalho, vigentes na maioria dos serviços de emergência da rede pública, o que tem gerado situações de franca hostilidade por parte dos pacientes e familiares.

Alguns estímulos emocionais que acompanham o adoecer segundo Nogueira-Martins ${ }^{14}$ são:

$>\mathrm{O}$ contato íntimo e freqüente com a dor e o sofrimento;

$>$ lidar com a intimidade corporal e emocional;

$>0$ atendimento de pacientes terminais;

$>$ lidar com pacientes difíceis: queixosos, rebeldes e não aderentes ao tratamento, hostis, reivindicadores, auto-destrutivos, cronicamente deprimidos;

$>$ lidar com as incertezas e limitações do conhecimento médico e do sistema assistencial que se contrapõem às demandas e expectativas dos pacientes e familiares que desejam certezas e garantias.

Oitenta por cento dos médicos brasileiros consideram a atividade médica desgastante, sendo os principais fatores de desgaste: excesso de trabalho/multiemprego, baixa remuneração, más condições de trabalho, responsabilidade profissional, área de atuação/especialidade, relação médicopaciente, conflito/cobrança da população, perda da autonomia ${ }^{14}$.

Clever $^{8}$ aborda diversos aspectos do exercício profissional e apresenta alguns interessantes dados sobre o trabalho dos médicos:

$>$ Os médicos trabalham mais que a maioria das pessoas (15 horas por semana a mais que outros profissionais);

$>$ tiram menos tempo de férias (4 semanas/ano dos médicos X 8 semanas/ano de outros profissionais);

$>$ trabalham maior número de anos do que a população geral.

Um estudo realizado com profissionais de quatro núcleos de Saúde da Família do Centro de Saúde Escola da Faculdade de Medicina de Ribeirão Preto, Universidade de São Paulo, e em um núcleo do município de Ribeirão Preto, estado de São Paulo, nos meses de novembro e dezembro de 2001, constatou que $62 \%$ dos indivíduos apresentavam estresse. A predominância dos sintomas de estresse ocorreram na área psicológica (48\%), seguida da área física (39\%). Apenas 13\% dos trabalhadores apresentaram igualdade quanto às áreas de distribuição dos sintomas.

Em outro caso, um estudo preliminar para constatar a ocorrência de ansiedade e estresse em psiquiatras de um Hospital Geral Universitário, foi utilizada uma escala internacional para ansiedade de Hamilton. Os resultados mostraram que $89 \%$ dos entrevistados sentiam angústia, $78 \%$ por razões profissionais e razões extra-profissionais conjuntamente; $11 \%$ só por razões profissionais e $11 \%$ que não sentiam angústia. Vemos, portanto, que a maioria dos entrevistados associa a angústia ao trabalho e à satisfação profissional. A maioria das pesquisas sobre o estresse, ansiedade e angústia nos profissionais médicos giram sempre em torno da insatisfação profissional, dos problemas relacionados ao trabalho, das questões de relacionamento interpessoal, das carências financeiras, das sobrecargas de serviço, da tensão, do pouco treinamento, da insegurança e das solicitações e pressões ocupacionais, o resultado do estudo acima demonstrou que $72 \%$ dos entrevistados estão insatisfeitos com o trabalho e com o relacionamento com os colegas ${ }^{2}$.

O objetivo desse estudo foi verificar a prevalência de Burnout entre médicos plantonistas de um hospital público. 


\section{MÉTODOS}

Para a consecução dos objetivos foi escolhido um Hospital Público de São Paulo localizado na zona Oeste da cidade. Foi feito contato com cada colega médico e o convite para participar do estudo, nessa ocasião foi solicitado que os participantes assinassem o termo de consentimento livre e esclarecido de acordo com a resolução 196 do CRM de 10 de outubro de 1996.

Na seqüência foi aplicado em 76 médicos um questionário constando a $1^{\underline{a}}$ parte de dados sóciodemograficos e profissionais e a $2^{\underline{a}}$ parte do Maslach Burnout Inventory $(\mathrm{MBI})$.

O MBI de Maslach e Jackson ${ }^{13}$, é um questionário de auto-informação para ser respondido através de uma escala do tipo Likert de 7 pontos, indo de " 0 " como "nunca" a " 6 " como "todos os dias". Contém 22 itens, 9 são relativos à dimensão Exaustão Emocional (EE), 5 à Despersonalização (DE) e 8 à Realização Pessoal (RP). Vários estudos têm demonstrado as propriedades psicométricas do $\mathrm{MBI}$. Validação fatorial (itens de saturação acima de 0,40 ), consistência interna ( $E E=$ alfa de 0,90; $\mathrm{DE}=$ alfa de 0,79 e RP = alfa de 0,71 ), validação convergente, estudos com teste-reteste. Para a $3^{\text {a }}$ edição, em uma amostra de 3.727 , observaram alfas de Crombach na razão de 0,89 para EE, 0,80 para DE (ou cinismo como passou a ser denominada esta escala) e 0,76 para RP (ou eficiência profissional).

Foi construída uma planilha no Microsoft Office Excel 2003 Ink, onde foram lançados as respostas obtidas, e então, realizada a análise estatística descritiva dos questionários. Consideramos como Síndrome de Burnout os resultados que atingiram os níveis determinados pelas escalas do MBI, ou seja, alta exaustão emocional, baixa realização pessoal e alta despersonalização.

Quadro. Parâmetros NEPASB: notas de corte.

\begin{tabular}{|l|l|l|l|}
\hline $\begin{array}{l}\text { Pontos de corte } \\
\text { Dimensões }\end{array}$ & Alto & Médio & Baixo \\
\hline Exaustão Emocional (EE) & 26 & $16-25$ & 15 \\
Realização Pessoal (RP) & 43 & $34-42$ & 33 \\
Despersonalização (DE) & 9 & $3-82$ & \\
\hline
\end{tabular}

\section{RESULTADOS}

Dos 76 médicos analisados 17 eram pediatras, 15 clínicos, 14 cirurgiões, 7 anestesistas, 6 neonatologistas, 5 ortopedistas e 2 psiquiatras. Todos eram plantonistas com carga horária mínima de 12 horas diárias.
As características sócio-demográficas mostraram que $89 \%$ dos participantes se encontravam na faixa etária dos 26 aos 50 anos, destes $52 \%$ estavam entre os 36 e 50 anos. A faixa etária que apresentou maior número de indivíduos está entre os 26 e 30 anos, que representa $21 \%$ desta amostra. A média de idade ficou por volta dos 38 anos.

Neste estudo encontramos uma proporção maior de homens do que de mulheres, correspondendo o sexo masculino a $54 \%$ da amostra. Grande também foi a proporção de indivíduos casados correspondendo a $56 \%$ desta população estudada, enquanto os solteiros corresponderam a $38 \%$, separados $6 \%$ e viúvos $1 \%$. A média de médicos que tem filhos é quase a mesma dos que não tem, sendo de $51 \%$ e $49 \%$, respectivamente.

Tabela 1. Distribuição dos médicos num Hospital Estadual de São Paulo de acordo com os dados sóciodemográficos.

\begin{tabular}{l|l|l}
\hline $\begin{array}{l}\text { Características } \\
\text { Faixa Etária (N = 76) }\end{array}$ & $\mathbf{N}$ & $\%$ \\
\hline $26|-| 30$ & 17 & 21 \\
$31 \mid 35$ & 12 & 16 \\
$36|-| 40$ & 15 & 20 \\
$41 \mid 45$ & 09 & 12 \\
$46|-| 50$ & 15 & 20 \\
$51 \mid 55$ & 02 & 03 \\
$56 \mid-60$ & 04 & 05 \\
$61 \mid 65$ & 02 & 03 \\
\hline Sexo (N=76) & & \\
\hline Masculino & 41 & 54 \\
Feminino & 35 & 46 \\
\hline Estado Civil (N=76) & 29 & \\
\hline Solteiro(a) & 42 & 56 \\
Casado(a) & 04 & 06 \\
Separado(a) & 01 & 01 \\
Viúvo(a) & & \\
\hline Filhos (N = 76) & 39 & 51 \\
\hline Sim & 37 & 49 \\
\hline Não & & \\
\hline
\end{tabular}

O número de médicos que fizeram especialização era quase a totalidade atingindo $97 \%$ da amostra. E 89\% dos médicos eram formados há mais de 5 anos.

Os cargos que apresentaram maior número de participantes eram aqueles que se enquadram nas especialidades básicas como: cirurgião geral $18 \%$, clínico geral $20 \%$, pediatra $22 \%$, ginecologista $11 \%$, perfazendo $71 \%$ dos entrevistados.

A área do pronto socorro concentrava $61 \%$ dos médicos, o centro obstétrico $11 \%$ e o centro cirúrgico $9 \%$.

Saúde, Ética \& Justiça, São Paulo. 2006;11(1/2):29-35. 
Quanto à carga horária semanal, $47 \%$ dos médicos trabalhavam entre 61 e 90 horas e, $81 \%$ trabalhavam entre 30 e 90 horas. Outro dado importante é o número de empregos por médico, $52 \%$ tinham 4 ou mais empregos e $91 \%$ tinham mais de um (Tabela 2).

Tabela 2. Distribuição dos médicos num Hospital Estadual de São Paulo de acordo com as características profissionais.

\begin{tabular}{|c|c|c|}
\hline $\begin{array}{l}\text { Características } \\
\text { Escolaridade }(\mathrm{N}=76)\end{array}$ & $\mathbf{N}$ & $\%$ \\
\hline $\begin{array}{l}\text { Residência } \\
\text { Graduação }\end{array}$ & $\begin{array}{l}74 \\
02\end{array}$ & $\begin{array}{l}97 \\
03\end{array}$ \\
\hline \multicolumn{3}{|l|}{$\begin{array}{l}\text { Tempo de graduação em anos } \\
(N=72)\end{array}$} \\
\hline $\begin{array}{l}01|-| 05 \\
06|-| 10 \\
11|-| 15 \\
16|-| 20 \\
21|-| 25\end{array}$ & $\begin{array}{l}08 \\
20 \\
15 \\
20 \\
09\end{array}$ & $\begin{array}{l}11 \\
27 \\
21 \\
27 \\
13\end{array}$ \\
\hline \multicolumn{3}{|l|}{ Cargo $(\mathrm{N}=76)$} \\
\hline $\begin{array}{l}\text { Anestesiologista } \\
\text { Cirurgião Geral } \\
\text { Clínico } \\
\text { Ginecologista / Obstétra } \\
\text { Neonatologista } \\
\text { Ortopedista } \\
\text { Pediatra } \\
\text { Psquiatra }\end{array}$ & $\begin{array}{l}07 \\
14 \\
15 \\
08 \\
06 \\
05 \\
17 \\
04\end{array}$ & $\begin{array}{l}09 \\
18 \\
20 \\
11 \\
08 \\
07 \\
22 \\
05\end{array}$ \\
\hline \multicolumn{3}{|l|}{ Seção (N = 76) } \\
\hline $\begin{array}{l}\text { Pronto Socorro (Geral) } \\
\text { Psiquiatria } \\
\text { Centro Obstétrico } \\
\text { Berçário } \\
\text { U.T.I. / Pediátrica } \\
\text { U.T.I. / Adulto } \\
\text { Centro Cirúrgico }\end{array}$ & $\begin{array}{l}47 \\
04 \\
08 \\
06 \\
02 \\
02 \\
07\end{array}$ & $\begin{array}{l}61 \\
05 \\
11 \\
08 \\
03 \\
03 \\
09\end{array}$ \\
\hline \multicolumn{3}{|l|}{ Carga Horária Semanal $(\mathrm{N}=76)$} \\
\hline $\begin{array}{l}30 \text { a } 60 \text { horas } \\
61 \text { a } 90 \text { horas } \\
91 \text { a } 120 \text { horas } \\
\text { Mais de } 120 \text { horas }\end{array}$ & $\begin{array}{l}26 \\
35 \\
14 \\
01\end{array}$ & $\begin{array}{l}34 \\
47 \\
18 \\
01\end{array}$ \\
\hline \multicolumn{3}{|l|}{ Número de Empregos $(\mathrm{N}=76)$} \\
\hline $\begin{array}{l}01 \\
02 \\
03 \\
04 \text { ou mais }\end{array}$ & $\begin{array}{l}01 \\
10 \\
26 \\
39\end{array}$ & $\begin{array}{l}01 \\
13 \\
34 \\
52\end{array}$ \\
\hline
\end{tabular}

Nota-se que de acordo com os níveis da escala de Maslach, 74\% apontaram níveis entre moderados e altos de exaustão emocional, $28 \%$ demonstraram baixa realização pessoal e 79\% altos índices moderados a altos de despersonalização (Tabela 3).

Tabela 3. Distribuição dos médicos num Hospital Estadual de São Paulo de acordo com os resultados nas 3 escalas MBI.

\begin{tabular}{l|l|l}
\hline $\begin{array}{l}\text { Características } \\
\text { Exaustão Emocional }(\mathbf{n = 7 6 )}\end{array}$ & N & $\%$ \\
\hline Alto & 24 & 32 \\
Médio & 32 & 42 \\
Baixo & 20 & 26 \\
\hline Realização Pessoal $(\mathbf{n = 7 6 )}$ & & \\
\hline Alto & 27 & 36 \\
Médio & 28 & 36 \\
Baixo & 21 & 28 \\
\hline Despersonalização $(\mathbf{n = 7 6 )}$ & & \\
\hline Alto & 30 & 40 \\
Médio & 30 & 39 \\
Baixo & 16 & 21 \\
\hline
\end{tabular}

Encontramos um resultado de $11 \%$ dos médicos com a Síndrome de Burnout. 89\% não tem Burnout, (Tabela 4).

Tabela 4. Distribuição dos médicos num Hospital Estadual de São Paulo de acordo com o diagnóstico de "Burnout".

\begin{tabular}{l|l|l}
\hline Características & N & $\%$ \\
\hline Médicos com "Burnout" & 08 & 11 \\
Médicos sem "Burnout" & 68 & 89 \\
\hline TOTAL & 76 & 100 \\
\hline
\end{tabular}

\section{DISCUSSÃo}

Os achados deste estudo demonstraram que a maioria dos médicos avaliados se encontrava no período mais produtivo da vida que vai dos 26 aos 50 anos (totalizaram $89 \%$ ). Sendo que $52 \%$ destes estavam na faixa etária que comumente já se encontra com a carreira definida, ou seja, dos 36 aos 50 anos.

Hernandes ${ }^{12}$, em um estudo similar, encontrou uma idade média de 35,5 anos, onde a população participante somou um total de 287 profissionais da saúde.

A quase totalidade dos médicos (97\%) referiu alguma especialização, isto demonstra que se 
submeteram ao desgastante processo de residência médica antes de iniciarem suas carreiras, ficando mais expostos aos agentes estressores.

A grande maioria dos médicos referiu altas cargas horárias de trabalho, a ponto de $47,7 \%$, dos médicos entrevistados, trabalhar de 21 a 50 horas semanais a mais que a média da população geral do País, ou seja pelo menos $50 \%$ a mais que a média da população geral que fica em torno de 44 horas semanais conforme o que preconiza a CLT (Consolidação das Leis do Trabalho). O sobretrabalho, caracterizado pelo excessivo número de horas trabalhadas pelos médicos, as jornadas longas com poucas pausas para descanso e sem um lugar apropriado para isto, os poucos períodos de lazer e convívio familiar e o desgaste com o público têm exposto os médicos a implacáveis agentes agressores e podem estar contribuindo para os resultados acima da média de Exaustão Emocional. Este estudo demonstrou que a grande maioria dos diagnósticos de Síndrome de Burnout e de Exaustão Emocional se encontram na faixa de carga horária de trabalho entre 61 e 90 horas semanais.

O estudo também mostrou que a maioria dos médicos com síndrome de Burnout estava abaixo dos 35 anos, Maslach (1978) apud BenevidesPereira $^{3}$ atribuiu este fato a insegurança dos médicos ou do choque que sofrem diante a realidade do trabalho quando constatam que suas expectativas não possuem sustentação na realidade.

Os médicos que têm este perfil de altos níveis de EE e DP, estão geralmente entre a faixa etária de 31 aos 50 anos, nos cargos de clínico geral, pediatra, psiquiatra, anestesiologista, ginecologista, ortopedista, e especialmente em cirurgião geral.

O sexo que é mais acometido pela síndrome de Burnout, é o feminino. Benevides-Pereira ${ }^{3}$ relata que tem havido maior incidência da síndrome em mulheres, o que confirmamos em nosso estudo. Benevides-Pereira ${ }^{3}$ atribui este fato à facilidade das mulheres expressarem mais livremente suas emoções e se sensibilizarem mais facilmente com o problema alheio, trazendo sobre si sua própria carga de traumas e a dos outros a sua volta. Hernandez ${ }^{12}$ encontrou uma diferença de $13,8 \%$ a mais de casos de Burnout em mulheres em relação aos homens, no seu estudo.

Com relação ao estado civil, obtivemos uma surpresa ao comparar nosso trabalho com a literatura atual. Ocorreu maior número de doentes em indivíduos casados ( $75 \%$ do diagnóstico) representando $8 \%$ da população total. Para Benevides-Pereira ${ }^{3}$ os casados apresentam menos propensão a ter a Síndrome de Burnout, sendo os solteiros, viúvos e separados mais sujeitos à doença, o que não aconteceu em nosso estudo.

A presença de filhos tem sido apontada por Benevides-Pereira ${ }^{3}$ como um fator possibilitador de melhores estratégias de enfrentamento das situações conflito e dos agentes estressores, porém não foram encontradas diferenças significativas neste estudo feito em médicos. Neste trabalho encontramos um maior número de casos de Síndrome de Burnout em médicos que ainda não têm filhos, entretanto a diferença é de apenas $2 \%$ para os que têm filhos.

O tempo de graduação dos médicos variou entre 11 e 20 anos o que corresponde ao período de busca da estabilidade profissional, onde grandes mudanças já se tornam mais difíceis e concomitantemente as perspectivas de crescimento também se tornam mais raras.

Além disto, todos os médicos acometidos relatam ter três ou mais empregos, este fato por si só já inspira o Burnout, uma vez que levaria o médico a se desdobrar muito mais, indo de um serviço para outro, envolvendo trânsito, clima, contato com um número muito maior de pessoas e seus problemas, pressão quanto ao tempo e quanto a seus ganhos. Todos estes fatores tornam um pouco mais complicado para o médico se adaptar a sua rotina de trabalho, uma vez que é muito instável, ficando assim seus sistemas de defesa vulneráveis a ação dos agentes estressores.

Embora apenas $11 \%$ dos médicos tenham apresentado diagnóstico compatível com a Síndrome de Burnout, foram encontrados índices que chegam a quase $80 \%$ dos médicos com níveis médio e alto de Exaustão Emocional e Despersonalização que estariam muito próximos de desenvolver a síndrome.

Este estudo trata de uma pequena parcela de um grande problema, para que pudéssemos ter um quadro mais detalhado desta doença na população médica seria necessário um estudo mais amplo e aprofundado com um número de casos bem maior, com detalhamento das variáveis, bem como o levantamento de muitas outras que não foram questionadas.

\section{CONSIDERAÇÕES FINAIS}

Este estudo mostrou que o índice de Burnout em médicos só não é maior devido à existência de grande Realização Pessoal. Esta tem funcionado com um fator protetor, impedindo que os médicos desenvolvam a doença. Embora estejam submetidos a inúmeros estressores (sobretrabalho, vários empregos, rotina desgastante) eles gostam do seu trabalho e dele auferem grande auto-realização. 
Magalhães RAC, Glina DMR. Prevalence of Burnout in public hospital doctors in São Paulo. Saúde, Ética \& Justiça. 2006;11(1/2):29-35.

ABSTRACT: Objective: To verify the Burnout Syndrome in doctors at a Public Hospital in São Paulo city. Methods. A case study was conduced at a general public hospital in São Paulo. The application of Maslach Burnout Inventory (MBI) and social-demography inventory was applied in this cross-sectional study of 76 doctors (25\% of the total population). The MBI is an auto-applicable inventory composed of three scales: 1) emotional exhaustion, 2) personal fulfillment, and 3) despersonalization. Results and Discussion: Eleven percent (11\%) of the doctors had Burnout Syndrome: $47 \%$ were between 31 and 45 years old; $52 \%$ were female; $67 \%$ were married; $100 \%$ had completed postgraduate level education; and $34 \%$ were in the period between 11 to 25 years after post graduation. Of the cases, $25 \%$ were anesthetists, $25 \%$ were surgeons, $25 \%$ were pediatricians, $12.5 \%$ were bonesetters and clinicians, $62 \%$ were emergency room doctors and $37.5 \%$ worker in other parts of the hospital. $62.5 \%$ worked more than 61 hours per week and $100 \%$ had more than three jobs. Conclusion: Although only $11 \%$ of the doctors showed signs of burnout (high results in emotional exhaustion, despersonalization and low personal fulfillment), $74 \%$ had high emotional exhaustion and $79 \%$ had high despersonalization, the protective farctor being the high level of personal fulfillment.

KEY WORDS: Hospitalists. Pshysicians/psychology. Stress psychological/prevention \& control. Working conditions. Workplace/psychology.

\section{REFERÊNCIAS}

1. Ballone GJ. Estresse e trabalho. In: PsiqWeb Psiquiatria geral, última revisão, 2002. Estresse. Disponível em: http://www.psiq.med.br/cursos/ stress.html.

2. Ballone GJ, Ribeiro CS, Marchi R. A ansiedade em psiquiatras, 1988. Disponível em: http:// www.psiq.med.br/cursos/stress.html.

3. Benevides-Pereira AMT, organizador. Burnout: quando o trabalho ameaça o bem estar do trabalhador. São Paulo: Casa do Psicólogo; 2002.

4. Brasil. Conselho Nacional de Saúde. Resolução 196 de 10 de outubro de 1996. Disponível em: http:// www.cfm.org.br.

5. Brasil. Ministério da Saúde. Doenças relacionadas ao trabalho; manual de procedimentos para os serviços de saúde. Brasília (DF): Ministério da Saúde; 2001. Disponível em: http://www.inss.org.

6. Brasil. Consolidação das Leis do Trabalho - CLT. In: Vademecun Universitário de Direito 2004. São Paulo: Jurídica Brasileira; 2004.

7. Camelo $\mathrm{SHH}$, Angerami ELS. Sintomas de estresse em trabalhadores atuantes em cinco núcleos de saúde da família. Ribeirão Preto: Escola de Enfermagem de Ribeirão Preto, Universidade de São Paulo; 2004.

8. Clever LH. Tratado de medicina de Cecil-Loeb. Rio de Janeiro: Guanabara Koogan; 1990.

9. Ferreira $A B$ de H. Dicionário novo Aurélio século XXI. 3a. ed. Rio de Janeiro: Nova Fronteira; 1999.

10. Glina DMR, Rocha LE. Prevenção para a saúde no trabalho. In: Glina DMR, Rocha LE, organizadoras. Saúde mental no trabalho: desafios e soluções. São Paulo: Ed. VK; 2000. p.104.

11. Glina DMR. Fatores psicossociais e saúde do trabalhador. In: Aula ministrada no Curso de Especialização em Medicina do Trabalho, na Disciplina de Saúde Mental do Trabalho, da Faculdade de Medicina de São Paulo. São Paulo; 2005. p.16-7. [apostila].

12. Hernandez JR. Estrés y Burnout en profesionales de la salud de los niveles primário y secundário de atención. Rev Cubana Salud Pública. 2003;29(2):103-10.

13. Maslach C, Jackson S. The Malasch Burnout Inventary. Palo Alto, Calif.: Consulting Psycologist Press; 1981/ 1986.

14. Nogueira-Martins LA. Atividade médica: fatores de risco para a saúde mental do médico. Rev Bras Clin Terap. 1991;20:355-64.

15. Organização Mundial de Saúde. CID-10 Código Internacional de Classificação de Doenças: Capítulo F: transtornos mentais e do comportamento. São Paulo: EDUSP; 1996.

16. Pereira AMTB. Debate jurídico no Brasil sobre saúde mental e trabalho. 2004. Disponível em: http:// www.saudeepsicologia.com.br 